\title{
646.
}

\section{ON THE GENERAL EQUATION OF DIFFERENCES OF THE SECOND ORDER.}

[From the Quarterly Journal of Pure and Applied Mathematics, vol. xIv. (1877), pp. 23-25.]

CONSIDER the equation of differences

viz. we have

$$
u_{x}=a_{x-1} u_{x-1}+b_{x-2} u_{x-2},
$$

$$
\begin{aligned}
& u_{2}=a_{1} u_{1}+b_{0} u_{0}, \\
& u_{3}=a_{2} u_{2}+b_{1} u_{1}, \\
& u_{4}=a_{3} u_{3}+b_{2} u_{2}, \\
& u_{5}=a_{4} u_{4}+b_{3} u_{3}, \\
& u_{6}=a_{5} u_{5}+b_{4} u_{4},
\end{aligned}
$$

\&c.,

and thence

$$
\begin{aligned}
& u_{3}=\left|\begin{array}{c}
a_{2} a_{1} \\
+b_{1}
\end{array}\right| u_{1}+\quad a_{2} b_{0} u_{0},
\end{aligned}
$$

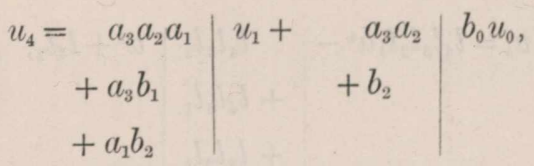

$$
\begin{aligned}
& u_{5}=a_{4} a_{3} a_{2} a_{1}\left|u_{1}+a_{4} a_{3} a_{2}\right| b_{0} u_{0}, \\
& +a_{4} a_{3} b_{1} \quad+a_{4} b_{2} \\
& +a_{4} a_{1} b_{2} \quad+a_{2} b_{3} \\
& +a_{2} a_{1} b_{3} \\
& +b_{1} b_{3}
\end{aligned}
$$




$$
\begin{aligned}
& u_{6}=a_{5} a_{4} a_{3} a_{2} a_{1}\left|u_{1}+a_{5} a_{4} a_{3} a_{2}\right| b_{0} u_{0}, \\
& +a_{5} a_{4} a_{3} b_{1} \quad+a_{5} a_{4} b_{2} \\
& +a_{5} a_{4} a_{1} b_{2} \quad+a_{5} a_{2} b_{3} \\
& +a_{5} a_{2} a_{1} b_{3} \quad+a_{3} a_{2} b_{4} \\
& +a_{3} a_{2} a_{1} b_{4} \quad+b_{4} b_{2} \\
& +a_{5} b_{3} b_{1} \\
& +a_{3} b_{4} b_{1} \\
& +a_{1} b_{4} b_{2}
\end{aligned}
$$

$\& c$.

It is now easy to see the law; viz. writing for instance

$$
u_{6}=54321 \cdot u_{1}+5432 \cdot b_{0} u_{0} \text {, }
$$

then 54321 has a leading term $a_{5} a_{4} a_{3} a_{2} a_{1}$ : it has terms derived from this by changing any pair $a_{2} a_{1}$ into $b_{1}, a_{3} a_{2}$ into $b_{2}, a_{4} a_{3}$ into $b_{3}, a_{5} a_{4}$ into $b_{4}$ : it has terms derived by changing any two pairs $a_{4} a_{3}, a_{2} a_{1}$ into $b_{3} b_{1} ; a_{5} a_{4}, a_{2} a_{1}$ into $b_{4} b_{1} ; a_{5} a_{4}, a_{3} a_{2}$ into $b_{4} b_{2}$, and so on; where observe that the expression a pair denotes the product of two consecutive $a$ 's.

And, similarly, 5432 has a leading term $a_{5} a_{4} a_{3} a_{2}$; the other terms being derived from this in the same manner precisely.

The solution of $u_{x}=l x\left(a u_{x-1}-u_{x-2}\right)$ is included in, and might be deduced from the foregoing, but it is convenient to obtain it separately. Supposing for greater simplicity that $u_{-1}=0, u_{0}=1$ (or, what is the same thing, $u_{0}=1, u_{1}=l_{1} \alpha$ ), then we find

$$
\begin{aligned}
& \begin{array}{l}
u_{0}=1, \\
u_{1}=l_{1} a, \\
u_{2}=l_{2} l_{1} a^{2}-l_{2}, \\
u_{3}=l_{3} l_{2} l_{1} a^{3}-\left|\begin{array}{r}
l_{3} l_{2} \\
+l_{3} l_{1}
\end{array}\right| a
\end{array} \\
& \begin{array}{c}
u_{4}=l_{4} l_{3} l_{2} l_{1} a^{4}-\left|\begin{array}{r}
l_{4} l_{3} l_{2} \\
+l_{4} l_{3} l_{1} \\
+l_{4} l_{2} l_{1}
\end{array}\right| a^{2}+l_{4} l_{2}, \\
u_{5}=l_{5} l_{4} l_{3} l_{2} l_{1} a^{5}-\left|\begin{array}{r}
l_{5} l_{4} l_{3} l_{2} \\
+l_{5} l_{4} l_{3} l_{1} \\
+l_{5} l_{4} l_{2} l_{1} \\
+l_{5} l_{3} l_{2} l_{1}
\end{array}\right| a^{3}+\left|\begin{array}{r}
l_{5} l_{4} l_{2} \\
+l_{5} l_{3} l_{2} \\
+l_{5} l_{3} l_{1}
\end{array}\right| a,
\end{array}
\end{aligned}
$$

\&c., 
viz. we may for example write

where

$$
u_{5}=l_{5} 4321 \cdot a^{5}-4321(\cdot) a^{3}+4321(:) a ;
$$

4321 denotes $l_{4} l_{3} l_{2} l_{1}$ :

in $4321(\cdot)$, we omit successively each number, viz. we thus obtain

$$
\begin{array}{r}
432+431+421+321, \\
=l_{4} l_{3} l_{2}+l_{4} l_{3} l_{1}+l_{4} l_{2} l_{1}+l_{3} l_{2} l_{1}:
\end{array}
$$

in 4321 (:), we omit successively each two non-consecutive numbers, viz. the omitted numbers being 1,$3 ; 1,4 ; 2$, 4 , we obtain

$$
\begin{aligned}
& 42+32+31, \\
= & l_{4} l_{2}+l_{3} l_{2}+l_{3} l_{1}:
\end{aligned}
$$

and so on, the omissions being each three numbers, each four numbers, \&c., no two of them being consecutive; thus in $654321(\therefore)$, the omissions are $5,3,1$, and $6,4,2$; or the symbol is

$$
\begin{array}{r}
642+531, \\
=l_{6} l_{4} l_{2}+l_{5} l_{3} l_{1} .
\end{array}
$$

As an application, a solution of the differential equation $\frac{d}{d x}\left(x \frac{d y}{d x}\right)+(x-a) y=0$ is $y=u_{0}+u_{1} x+u_{2} x^{2}+\&$ c., where $n^{2} u_{n}=a u_{n-1}-u_{n-2}$, and in particular $1^{2} u_{1}=a u_{0}$; the equation of differences is thus of the form in question, and retaining $l_{n}$ in place of its value, $=n^{2}$, the solution is $u_{0}=1, u_{1}=l_{1} a, u_{2}=l_{2} l_{1} a^{2}-l_{2}$, \&c. ut suprà. The differential equation was considered by the Rev. H. J. Sharpe, who mentioned it to Prof. Stokes. 